\title{
Genome scan for loci regulating HDL cholesterol levels in Finnish extended pedigrees with early coronary heart disease
}

\author{
Tiia Kangas-Kontio ${ }^{1,5}$, Sakari Kakko ${ }^{\star 1,5}$, Minna Tamminen ${ }^{1}$, Peter von Rohr ${ }^{2}$, Ina Hoeschele ${ }^{2}$, Tatu Juvonen ${ }^{3}$, \\ Juha Kere $^{4}$ and Markku J Savolainen ${ }^{1}$
}

Coronary heart disease (CHD) is the leading cause of mortality in Western societies. Its risk is inversely correlated with plasma high-density lipoprotein cholesterol (HDL-C) levels, and approximately $50 \%$ of the variability in these levels is genetically determined. In this study, the aim was to carry out a whole-genome scan for the loci regulating plasma HDL-C levels in 35 well-defined Finnish extended pedigrees ( 375 members genotyped) with probands having low HDL-C levels and premature CHD. The additive genetic heritability of HDL-C was $43 \%$. A variance component analysis revealed four suggestive quantitative trait loci (QTLs) for HDL-C levels, with the highest LOD score, 3.1, at the chromosomal locus 4p12. Other suggestive LOD scores were 2.1 at $2 \mathrm{q} 33,2.1$ at $6 \mathrm{p} 24$ and 2.0 at $17 \mathrm{q} 25$. Three suggestive loci for the qualitative low HDL-C trait were found, with a nonparametric multipoint score of 2.6 at the chromosomal locus $10 \mathrm{p} 15.3,2.5$ at $22 \mathrm{q} 11$ and 2.1 at $6 \mathrm{p} 12$. After correction for statin use, the strongest evidence of linkage was shown on chromosomes 4p12, 6p24, 6p12, 15q22 and 22q11. To search for the underlying gene on chromosome 6 , we analyzed two functional and positional candidate genes (peroxisome proliferatoractivated receptor-delta (PPARD), and retinoid X receptor beta, (RXRB)), but found no significant evidence of association. In conclusion, we identified seven chromosomal regions for HDL-C regulation exceeding the level for suggestive evidence of linkage.

European Journal of Human Genetics (2010) 18, 604-613; doi:10.1038/ejhg.2009.202; published online 25 November 2009

Keywords: atherosclerosis; complex trait; lipoprotein; linkage analysis

\section{INTRODUCTION}

Atherosclerosis is the primary cause of coronary heart disease (CHD) and stroke, accounting for approximately $50 \%$ of all deaths in Western societies. ${ }^{1}$ Plasma high-density cholesterol (HDL-C) levels are inversely related with the risk of $\mathrm{CHD},{ }^{2}$ and low plasma HDL-C is the most common dyslipidemia associated with premature and familial $\mathrm{CHD}^{3}$ Genetic factors account for approximately $50 \%$ of the variability in plasma HDL-C levels, and the inheritance of HDL-C levels is complex, being influenced by multiple genetic and environmental factors and by interactions between these factors. ${ }^{4}$ So far, genome-wide scans for the loci regulating HDL-C levels have been published with significant results reported with respect to 18 chromosomes (linkage studies are summarized in Table $1 a^{5-25}$ and genome-wide association studies in Table $1 b,{ }^{26-32}$ ) which is indicative of high genetic heterogeneity in HDL-C regulation. This genetic heterogeneity also involves the general link between low plasma HDL-C levels and increased CHD risk, as some genetic variants are associated with low HDL-C levels and decreased CHD risk (eg, apoA-I (Milano) $)^{33}$ and some with high HDL-C levels and increased $\mathrm{CHD}$ risk (eg, a hepatic lipase gene variant). ${ }^{34}$ To reduce the heterogeneity, we collected the sample based on probands with low plasma HDL-C levels and premature CHD, and other CHD risk factors were reduced by allowing only normal or moderately elevated levels of triglycerides (TG) and total cholesterol and no individuals with diabetes in the probands. The Finns originate from small founder populations sharing a relatively homogeneous gene pool and environment, ${ }^{35}$ both of which are helpful for the mapping of susceptibility genes.

The aim of this study was to search the whole genome for loci regulating plasma HDL-C levels in the population of Northern Finland, where there is a high prevalence of CHD. On the basis of the close relationship between CHD and low HDL-C, identification of the genes affecting HDL-C levels could lead to the discovery of major genetic factors involved in atherosclerosis susceptibility. To elucidate the genetics of the low HDL-C-trait associated with CHD, linkage analyses for quantitative trait loci (QTLs) regulating plasma HDL-C levels and loci linked to the qualitative low HDL-C trait were performed in 35 Finnish extended pedigrees with premature CHD and low HDL-C levels (644 individuals, 375 genotyped).

\section{SUBJECTS AND METHODS}

Subjects studied

Probands with premature CHD (ie, acute myocardial infarction, a coronary artery bypass graft operation or percutaneous transluminal coronary angio-

\footnotetext{
${ }^{1}$ Institute of Clinical Medicine, Department of Internal Medicine and Biocenter Oulu, University of Oulu and Clinical Research Center, Oulu University Hospital, Oulu, Finland; ${ }^{2}$ Virginia Bioinformatics Institute and Department of Statistics, Virginia Polytechnic Institute and State University, Blacksburg, VA, USA; ${ }^{3}$ nstitute of Clinical Medicine, Department of Surgery, University of Oulu, Oulu, Finland; ' ${ }^{4}$ Department of Medical Genetics, University of Helsinki and Department of Biosciences at Novum, Karolinska Institutet, Stockholm, Sweden

*Correspondence: Dr S Kakko, Department of Internal Medicine, University of Oulu, PO BOX 5000, FIN-90014 Oulu, Finland

Tel: +358 8315 4570; Fax: +358 8315 4543; E-mail sakari.kakko@oulu.fi

5 These authors equally contributed to this work.

Received 15 April 2009; revised 6 October 2009; accepted 13 October 2009; published online 25 November 2009
} 
Table 1a Summary of the loci for HDL-C in published genome-wide linkage scans

\begin{tabular}{|c|c|c|c|}
\hline Location & Subjects & $n$ & Article (reference) \\
\hline $1 p^{a}$ & White sib-ships with hypertension & 691 & Kullo et al, $2005^{5}$ \\
\hline $2 q 21-22$ & Framingham Heart Study families & 1294 & Arya et al, $2003^{6}$ \\
\hline $3 p 14$ & Four extended FCHL pedigrees & 198 & Gagnon et al, $2005^{7}$ \\
\hline $3 q 26$ & Asians with hypertension & 1228 & Bielinski et al, $2006^{8}$ \\
\hline $4 q 31.21$ & French Canadian Low HDL-C Families & 362 (13 families) & Dastani et al, $2006^{9}$ \\
\hline $4 q 34^{b}$ & Healthy adult nuclear families from the Victorian Family Heart Study & 274 sibling pairs & Harrap et al, $2006^{10}$ \\
\hline $5 p 14$ & NHLBI Family Heart Study pedigrees & 1027 (101 families) & Peacock et al, $2001^{11}$ \\
\hline $6 p 12-6 q 12$ & FH-kindred with hyperalphalipoproteinemia & $\begin{array}{l}34 \text { (1 multigenerational } \\
\text { kindred) }\end{array}$ & $\begin{array}{l}\text { Canizales-Quinteros } \\
\text { et al, } 2003^{12}\end{array}$ \\
\hline $6 q 23^{c}$ & Framingham Heart Study families & 907 & Yang et al, $2005^{13}$ \\
\hline $6 q 23-24^{d}$ & Framingham Heart Study families & 1294 & Arya et al, $2003^{6}$ \\
\hline $7 q 31$ & Sibling pairs with type 2 diabetes mellitus from Ghana and Nigeria & $\begin{array}{l}295 \text { affected sibling } \\
\text { pairs }\end{array}$ & Adeyemo et al, $2005^{14}$ \\
\hline $8 q 23$ & Finnish low-HDL and FCHL patients & $\begin{array}{c}\text { Low HDL: } 219 \text { (25 } \\
\text { families) FCHL } 139 \text { (29 } \\
\text { families) }\end{array}$ & Soro et al, $2002^{15}$ \\
\hline $8 q 24^{d}$ & Mexican Americans, families randomly ascertained (subsample of the SAFHS) & 477 (10 pedigrees) & Almasy et al, $1999^{16}$ \\
\hline $9 p$ & Mexican Americans from the San Antonio Family Diabetes Study & 415 (27 families) & Arya et al, $2002^{17}$ \\
\hline $10 q 11$ & Finnish FCHL and low-HDL patients & 1109 (92 families) & Lilja et al, $2004^{18}$ \\
\hline $12 q 14.1$ & $\begin{array}{l}\text { The Québec Family Study, randomly ascertained families and families with obese } \\
\text { probands }\end{array}$ & 930 & Bosse et al, $2004^{19}$ \\
\hline $12 q$ & $\begin{array}{l}\text { Chinese subjects (in the Stanford-Asian Pacific Program in Hypertension and Insulin } \\
\text { Resistance study) }\end{array}$ & 1538 (509 families) & Hsiao et al, $2006^{20}$ \\
\hline $12 q 23-24^{e}$ & Healthy white families from the United States and Canada & 99 families & Feitosa et al, $2006^{21}$ \\
\hline $12 q 24$ & Asians with hypertension & 1228 & Bielinski et al, $2006^{8}$ \\
\hline $15 q 21$ & NHLBI Family Heart Study pedigrees & 3408 & Feitosa et al, $2007^{22}$ \\
\hline $15 q 22$ & Turkish and Mediterranean families with metabolic syndrome (in the GEMS Project) & 770 (60 families) & Yu et al, $2005^{23}$ \\
\hline $15 q 22^{f}$ & Mexican Americans, families randomly ascertained (subsample of the SAFHS) & 477 (10 pedigrees) & Almasy et al, $1999^{16}$ \\
\hline $16 q 22-24$ & $\begin{array}{l}\text { Mexican American probands, families randomly ascertained (subsample of the SAFHS, } \\
\text { diabetics included) }\end{array}$ & 472 (10 pedigrees) & Mahaney et al, $2003^{24}$ \\
\hline $16 q 24.1$ & Dutch and Finnish FCHL patients and Finnish low-HDL patients & $\begin{array}{c}\text { FCHL: } 341 \text { (48 } \\
\text { families), low HDL } 219 \\
\text { (25 families) }\end{array}$ & Pajukanta et al, $2003^{25}$ \\
\hline
\end{tabular}

Abbreviations: HDL-C, high-density lipoprotein cholesterol; FCHL, familial combined hyperlipidemia; NHLBI, National Heart, Lung, and Blood Institute; FH, familial hypercholesterolemia; SAFHS, San Antonio Family Heart Study.

Only results with an LOD score $\geqslant 3.0$ are shown.

${ }^{5}$ Bivariate analysis for HDL-C and TG.

aBivariate analysis for HDL-C and LDL particle size.

${ }^{\mathrm{b}} \mathrm{Z}$-score $=3.9$.

${ }^{\mathrm{C}} \mathrm{HDL}_{3}-\mathrm{C}$ as a trait.

dBivariate analysis (In BMI-In HDL-C).

eBivariate analysis for HDL-C and TG.

${ }^{\mathrm{f}} \mathrm{HDL} 2 \mathrm{a}_{\mathrm{a}} \mathrm{C}$ as a trait.

plasty before the age of 55 years) were selected from the records of Oulu University Hospital. In addition, the probands were required to have low HDL-C levels $(<1.1 \mathrm{mmol} / \mathrm{l})$ and normal to moderately elevated levels of TG $(<3.5 \mathrm{mmol} / \mathrm{l})$ and total cholesterol $(<7 \mathrm{mmol} / \mathrm{l})$, no diabetes and an entry in the hospital records indicating a family history of CHD. In addition, all the relatives of the proband (independent of their CHD status and HDL-C levels) who were willing to participate were examined and extended pedigrees were recruited to ascertain the genetic background. The total number of family members was 644 (with 375 subjects genotyped). There were 35 pedigrees with three generations on average (minimum 2 and maximum 5) and with an average pedigrees size of 19 subjects (minimum 5 and maximum 89). Blood samples for DNA extraction and lipid measurement were obtained from each subject, and lipid measurements were performed in the case of the CHD patients before or at least 3 months after myocardial infarction or coronary bypass operation. Information about medication, past medical history and smoking (recoded as current smoker or not and in pack-years of smoking and the number of cigarettes smoked currently) was elicited using a questionnaire. Altogether, 40 of the subjects were receiving statin therapy. The daily doses of statin taken by our subjects (collected between 1993 and 2000, before the era of intensive statin treatment) were small (simvastatin $10 \mathrm{mg} /$ day 20 subjects, simvastatin $20-30 \mathrm{mg} /$ day 3 subjects, lovastatin $\max 40 \mathrm{mg} /$ day 6 subjects, fluvastatin $\max 40 \mathrm{mg} /$ day 5 subjects, atorvastatin $\max 20 \mathrm{mg} /$ day 3 subjects and pravastatin max $20 \mathrm{mg} /$ day 3 subjects), and their effect on plasma HDL-C levels was expected to be only modest (an increase of $<6 \%$ ), ${ }^{36}$ so that these subjects were not excluded from the analysis. However, to control the statin use as a possible confounding factor, the chromosomes revealing an LOD/NPL score $>1.5$ were re-analyzed with a constant $(6 \%$ of the mean HDL-C levels: $0.06 \mathrm{mmol}$ for men and $0.09 \mathrm{mmol}$ for women) subtracted from HDL-C values of the statin users. ${ }^{37}$ The study was approved by the ethical committee of the University of Oulu, and all the subjects gave their written informed consent.

\section{Lipid and lipoprotein measurements}

Blood samples were obtained after an overnight fast. Plasma lipoprotein fraction were separated using sequential ultracentrifugation and concentrations of cholesterol and TG in the plasma and lipoprotein fractions were determined using enzymatic colorimetric methods, as described in detail earlier. ${ }^{38}$ 
Table 1b Summary of the loci for HDL-C in published genome-wide association studies

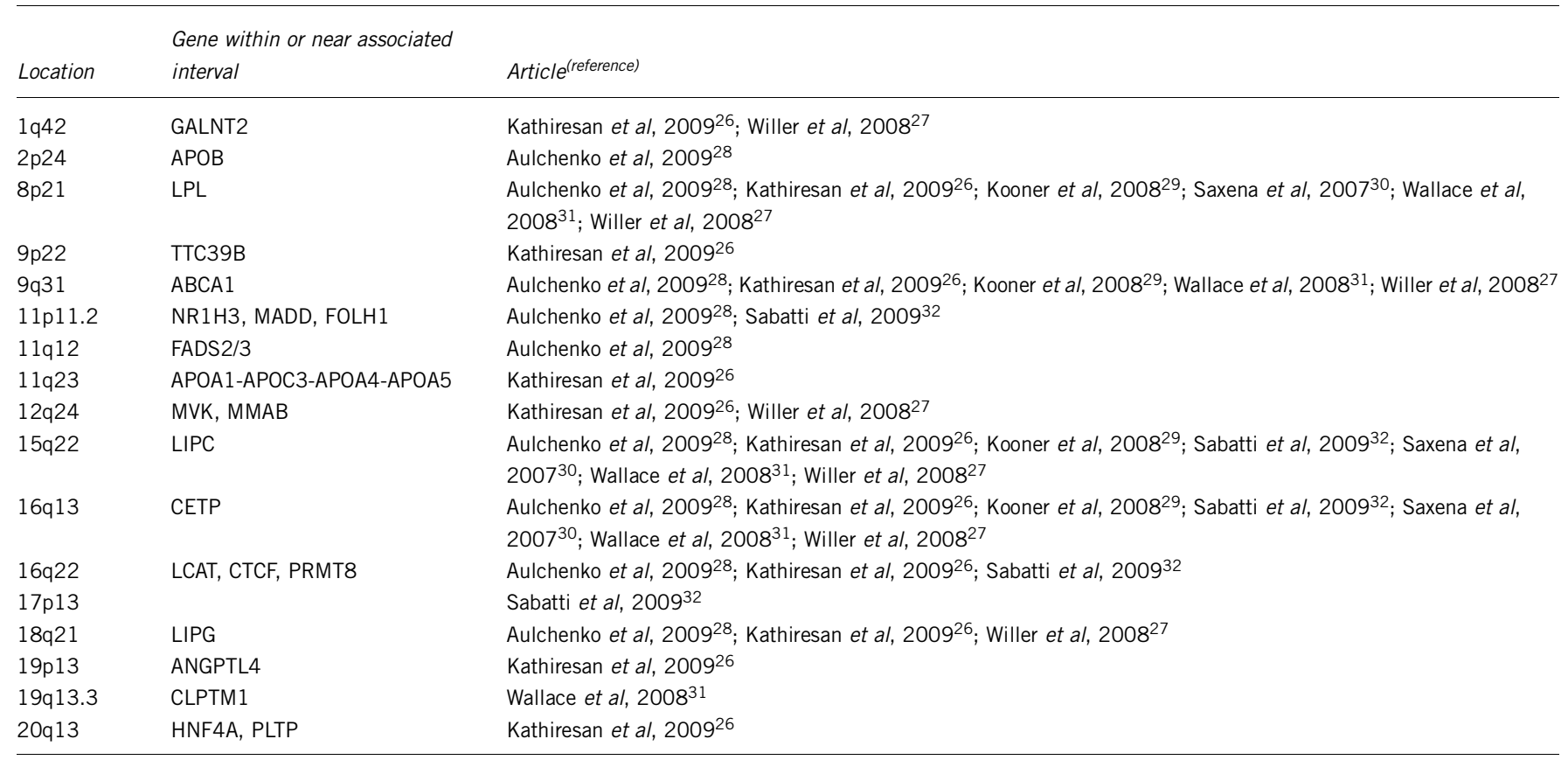

Abbreviations: GALNT2, GalNAc transferase 2; APOB, apolipoprotein B; LPL, lipoprotein lipase; TTC39B, tetratricopeptide repeat protein 39B; ABCA1, ATP-binding cassette subfamily A member 1; NR1H3, nuclear receptor subfamily1 group H member 3; MADD, map kinase-activating death domain; FOLH1, folate hydrolase 1; FADS2/3, fatty acid desaturase 2, 3; APOA1-APOC3-APOA4-APOA5, apoliprotein A-1, C-3, A-4 and A-5; MVK, mevalonate kinase; MMAB, cobalamin adenosyltransferase; LIPC, hepatic lipase; CETP, cholesteryl ester transfer protein; LCAT, lecithin:cholesterol acyltransferase; CTCF, CCCTC-binding factor; PRMT8, protein arginine methyltransferase 8; LIPG, endothelial lipase; ANGPTL4, angiopoietin-like 4; CLPTM1 cleft lip and palate-associated transmembrane protein 1; HNF4A, hepatocyte nuclear factor 4-alpha; PLTP, phospholipid transfer protein.

All the analyses used population-based cohorts or cohorts with cardiovascular disease-related traits. Most of the studies used combinations of several study samples. The number of subjects in the initial genome-wide scans ranged between 1955 and 21412 subjects.

\section{DNA analysis}

The ABI Prism Linkage Mapping Set 1 (Applied Biosystems, Foster City, CA, USA) was used for genotyping microsatellite markers in the initial screening. The set consisted of 358 markers covering the whole genome (except the $\mathrm{Y}$ chromosome) at about $10 \mathrm{cM}$ resolution. The markers were originally selected from the 1996 Genethon map. ${ }^{39}$ Additional markers on chromosomes 2, 4, 6 , 10,15 and 22 were selected for the regions showing highest evidence of linkage. Genomic DNA extraction and the microsatellite marker genotyping with the ABI 377 and ABI 3100 automatic sequencers were performed as described earlier ${ }^{40}$ and recommended by the manufacturer (Applied Biosystems). The sizes of the alleles were determined using the Genescan (Version 3.1), Genotyper (2.0) (with ABI 377) and GeneMapper (3.5) (with ABI 3100) programs. A total of eight SNPs (shown in Table 4) on two functional candidate genes, peroxisome proliferator activated receptor delta (PPARD) and retinoid X receptor beta $(R X R B)$, were selected from the HapMap to cover all the haploblocks of the genes. The genes were located on chromosome 6 near the only region showing suggestive evidence of linkage in both qualitative and quantitative linkage analysis. The SNPs were genotyped using TaqMan SNP Genotyping Assays and the Applied Biosystems 7000 Real-Time PCR System (Applied Biosystems).

\section{Statistical analysis}

Pedigree integrity was checked by GRR (graphical representation of relationships $)^{41}$ using IBS allele sharing to detect pedigree errors, as pairs of different classes of relatives and non-relatives can be characterized by the unique distribution of allele shared across the genome. Mendelian errors were checked with the PedCheck program. ${ }^{42}$

\section{Quantitative linkage analysis}

A multipoint variance components linkage analysis was used to test linkage between the marker loci and HDL-C, which was based on specifying the expected genetic covariances between pairs of relatives as a function of their identity by descent (IBD) at a marker linked to a QTL. ${ }^{43}$ The total observed phenotypic variance was split into components attributable to QTL, residual polygenic effects and non-genetic effects.

The two-point and multipoint analyses were performed with the SOLAR (Sequential Oligogenic Linkage Analysis Routines) program, ${ }^{44}$ version 4.0.7 (Copyright (C) 1995-2009, Southwest Foundation for Biomedical Research, http://solar.sfbrgenetics.org/), using variance components analysis for extended pedigrees. Multipoint identity-by-descent matrices were estimated using Markov chain Monte Carlo methods implemented in SimWalk2 http://www. genetics.ucla.edu/software/simwalk ${ }^{45}$ (with direct SOLAR support). The presence of a putative QTL was tested by means of a likelihood ratio statistic (LOD score). Plasma HDL-C levels were used as a continuous variable in the analysis, and the sex, age and body mass indices of the subjects (BMI, only when stated), being statistically significant covariates in the data set $(P<0.001)$, were also considered in the analysis. Ascertainment correction by conditioning for the probands was performed. To deal with skewness and kurtosis, log transformation and LOD adjustment were used, the latter using a simulation to build up the distribution of LOD scores that one could expect to observe under the null hypothesis of no linkage. This consisted of 10000 trials, in each of which a fully informative marker completely unlinked to the trait was simulated and trait linkage was then tested at that simulated marker. The LOD adjustment regresses the observed LOD scores against the LOD scores expected for a multivariate normal trait. The inverse of the slope of the regression line is the LOD adjustment. Empirical $P$-values for the LOD scores in the sample were also determined using the pedigree data by simulation of 10000 replicates of a fully informative marker completely unlinked to the trait.

\section{Qualitative linkage analysis}

For the qualitative non-parametric linkage (NPL) analysis, subjects having their measured HDL-C levels in the lowest 10th percentile of the sex-specific population HDL-C levels (distributions taken from the control cohort of the OPERA study ${ }^{38}$ ) were coded as being affected. This limit was $1.08 \mathrm{mmol} / \mathrm{l}$ for 
Table 2 Characteristics of all the genotyped subjects (quantitative analysis sample) and of the subset of the subjects used for the analysis of low HDL-C trait (qualitative analysis sample)

\begin{tabular}{|c|c|c|c|c|}
\hline & \multicolumn{2}{|c|}{ Quantitative analysis sample $e^{a}$} & \multicolumn{2}{|c|}{ Qualitative analysis sample ${ }^{\mathrm{b}}$} \\
\hline & Men & Women & Men & Women \\
\hline Number of subjects & 212 & 163 & 137 & 104 \\
\hline Age & $48.6(13.1)$ & $49.3(16.5)$ & $48.0(13.9)$ & $46.3(17.3)$ \\
\hline CHD patients & $119(56 \%)$ & $34(21 \%)$ & $60(44 \%)$ & $12(12 \%)$ \\
\hline Age at first $\mathrm{CHD}$ event & $48.8(10.1)$ & $52.9(9.7)$ & $49.5(10.0)$ & $54.5(10.2)$ \\
\hline Low HDL-C patients ${ }^{c}$ & $55(26 \%)$ & $29(18 \%)$ & $49(36 \%)$ & $24(23 \%)$ \\
\hline Body mass index $\left(\mathrm{kg} / \mathrm{m}^{2}\right)$ & $26.9(3.9)$ & $25.5(4.7)$ & $26.5(3.9)$ & $25.1(5.1)$ \\
\hline Total cholesterol (mmol/l) & $5.37(1.06)$ & $5.49(1.15)$ & $5.44(1.15)$ & $5.56(1.25)$ \\
\hline HDL cholesterol (mmol/l) & $1.07 *(0.29)$ & $1.39(0.33)$ & $1.00 *(0.28)$ & $1.35(0.35)$ \\
\hline LDL cholesterol (mmol/l) & $3.33(0.87)$ & $3.30(0.97)$ & $3.38(0.95)$ & $3.36(1.07)$ \\
\hline Triglycerides (mmol/l) & $1.78(1.02)$ & $1.49(1.54)$ & $1.90(1.14)$ & $1.55(1.88)$ \\
\hline Current smokers & $49(23 \%)$ & $23(14 \%)$ & $34(25 \%)$ & $12(12 \%)$ \\
\hline Lipid-lowering drug in use & $34(16 \%)$ & $6(4 \%)$ & $21(15 \%)$ & $3(3 \%)$ \\
\hline
\end{tabular}

Abbreviations: $\mathrm{HDL}-\mathrm{C}$, high-density lipoprotein cholesterol; $\mathrm{CHD}$, coronary heart disease.

Values shown for the subjects genotyped are expressed as means (SD) or numbers of subjects (percentages).

Values shown for the subjects genotyped are

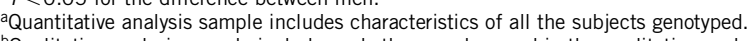

${ }^{b}$ Qualitative analysis sample includes only the samples used in the qualitative analysis of the low HDL-C trait.

'Subjects having their measured HDL-C levels in the lowest 10th percentile for the general population were coded as affected.

women and $0.864 \mathrm{mmol} / \mathrm{l}$ for men. Those with their measured plasma HDL-C levels over the lowest 10th percentile were coded as being unaffected. The sample for the qualitative analysis included only those pedigrees with at least two subjects affected according to the low HDL-C criterion (19 pedigrees, 388 individuals, 241 genotyped).

NPL analysis, also known as allele sharing statistics, is based on IBD measurements at the marker loci and is independent of specific models for the inheritance of the trait phenotype. If a marker is linked to a disease locus, one expects to see among the affected cases a clustering of a few marker alleles descended from the pedigree founders. The multipoint NPL analysis was carried out in this study using the SimWalk2 (version 2.83$)^{45}$ and Merlin (version 0.9.12b, http://www.sph.umich.edu/csg/abecasis/MERLIN/) ${ }^{46}$ programs. The allele frequencies for each marker were estimated from all individuals by the Downfreq program. ${ }^{47} \mathrm{Mega} 2{ }^{48}$ was used to construct all the input files for the SimWalk2 and Merlin programs. An exact analysis using the Lander-Green algorithm was performed by Merlin on the pedigrees of small or intermediate size ( 9 pedigrees) and the more complex pedigrees (10 pedigrees) were analyzed by SimWalk2 using Markov chain Monte Carlo and simulated annealing algorithms. SimWalk2 was used to combine the pre-computed scores for the smaller pedigrees with the estimates obtained for the large pedigrees and then to compute empirical $P$-values both for individual pedigrees and for the overall data set.

SimWalk2 presents the results of five statistics (BLOCKS, MAX-TREE, ENTROPY, NPL_PAIR and NPL_ALL) as empirical $P$-values and $-\log _{10}(P$-value) (NPL score), in which each statistic measures the degree of clustering of the founder alleles among the affected cases. BLOCKS is most powerful at detecting linkage to a recessive trait, MAX-TREE is the most powerful at detecting linkage to a dominant trait, ENTROPY is a measure of the entropy of the alleles among the affected cases, and NPL_PAIR and NPL_ALL are most powerful at detecting linkage to an additive trait and also the two most commonly used statistics incorporated in the most widely used software packages. ${ }^{49}$ NPL_ALL (used in the results when not otherwise reported) is a measure of whether a few founder alleles are over-represented in the affected cases and corresponds to the NPL-all statistic used in GeneHunter, Allegro, Mendel and Merlin, for example.

\section{Association analysis}

QTL association was tested with the Mendel program using variance component models, treating genotypes at the marker locus as predictors modifying the mean for a quantitative trait (QTL association, model 1). This 'measured genotype' approach controls for random environment and polygenic back- grounds while remaining in the frequentist domain of maximum likelihood estimation and likelihood ratio tests. ${ }^{50}$

All the calculations were performed on the computers of the Center for Scientific Computing, Espoo, Finland.

\section{RESULTS}

The characteristics of the whole sample and the subset of subjects used in the qualitative analysis are shown in Table 2 . The only statistically significant difference between these study samples was in HDL-C levels $(P<0.05)$, indicating the inclusion criteria for qualitative linkage analysis in which each family had to have at least two affected (low HDL-C phenotype) family members. CHD was diagnosed in $41 \%$ of our study subjects $(56 \%$ of the men and $21 \%$ of the women in the whole sample), and their relatively young age at CHD onset (49 and 53 years, respectively) emphasizes the potentially strong genetic component in CHD in our pedigrees. The mean plasma HDL-C levels of the sample $(1.07 \mathrm{mmol} / \mathrm{l}$ for men and $1.39 \mathrm{mmol} / \mathrm{l}$ for women) were lower than the levels reported for Northern-Finnish population (for men, 1.22 and for women, 1.56). ${ }^{38} \mathrm{CHD}$ patients had lower plasma HDL-C levels than the healthy subjects among both the men $(0.96 \mathrm{vs}$ $1.15 \mathrm{mmol} / \mathrm{l}, \quad P<0.001)$ and the women $(1.27$ vs $1.43 \mathrm{mmol} / \mathrm{l}$, $P=0.06$ ), suggesting the importance of HDL as a risk factor for CHD. Only 40 study subjects (ie $11 \%$ of the whole sample) used statins, as the sample was collected before the 'statin era'. The studied subjects were slightly overweight according to their average BMI (men 26.9, SD 3.9, women 25.5, SD 4.7).

\section{Quantitative linkage analysis}

The estimated additive genetic heritability of plasma HDL-C was $43 \%$ and the proportion of variance due to covariates (sex and age) was $23 \%$. On the basis of the empirical $P$-value estimate, if the hypothesis of no linkage was true, we would expect 4 out of 10000 observed LOD scores to be $\geqslant 3.1$, giving us an empirical $P$-value of 0.0004 (unadjusted) for the LOD score of 3.1 in our sample. With 10000 simulations, we found LOD scores $\geqslant 2.0$ nine times $\left(p_{\text {empirical }}=0.0009\right)$ and LOD scores $\geqslant 1.912$ times $\left(p_{\text {empirical }}=0.0012\right)$. To determine the level of suggestive evidence of linkage, we compared the unadjusted empirical $P$-values from the simulations with the 
point-wise levels calculated using the equation presented in the article of Lander and Kruglyak. ${ }^{51}$ The average crossover rate, given the types of relatives in our pedigrees, was 2.94. According to this, our threshold for suggestive evidence of linkage is $P=0.0011$ and when we compare it with the results of the simulations, we can conclude that an LOD score of 2.0 may be considered as suggestive evidence of linkage in our study.

The initial QTL scan, carried out on 35 pedigrees with sex and age as covariates, yielded multipoint LOD scores (MLOD) of over 1 on chromosomes 2 (MLOD of 1.6: peak between the markers D2S364 and D2S325), 4 (2.4: D4S405 and D4S428), 6 (1.5: D6S289 and D6S276), 15 (1.3: D15S117 and D15S153) and 17 (2.0: D17S784). In the second stage, new markers were genotyped (Figure 1a), and chromosomes 2, 4, 6 and 17 exceeded the level of suggestive evidence of linkage (LOD scores $\geqslant 2.0$ ) at chromosomal locations 2q33 (MLOD 2.1), 4p12 (two-point LOD 3.1, MLOD 2.6), 6p24 (two-point LOD 2.1) and 17q25 (MLOD 2.0) (Figure 2), whereas LOD score for chromosome 15 (MLOD 1.9) was narrowly under the level of suggestive linkage. Chromosomes with LOD scores $>2$ were also analyzed with all the significant covariates (sex, age and BMI). Inclusion of BMI did not alter the results, except in the case of chromosome 6 , in which the region $6 \mathrm{p} 24$ yielded a two-point LOD score of 2.7. In conclusion, chromosomal regions $2 \mathrm{q} 33,4 \mathrm{p} 12,6 \mathrm{p} 24$ and $17 \mathrm{q} 25$ showed suggestive evidence of linkage in the quantitative linkage analysis, the linkage on $4 \mathrm{p} 12$ being close to the level that is generally considered as significant evidence of linkage. The results are also summarized in Table 3.

\section{Qualitative linkage analysis}

The first stage of qualitative multipoint NPL analysis (Figure 1b) revealed one suggestive locus with an NPL score $\left(-\log _{10} P\right.$-value) of $2.6(P=0.003)$ at chromosomal locus 10p15.3 (close to the marker D10S249). For the regions in which the scores were $>1.5$, we selected nine new markers: five on chromosome 10, two on chromosome 6 and two on chromosome 22. In addition, four new families with 27 genotyped persons were added to the analysis. Thus, genotyping of the additional markers and families resulted in suggestive NPL scores of 2.1 on chromosome $6 \mathrm{p} 12,2.3$ on $10 \mathrm{p} 15$ and 2.5 on 22q11 (Figure 2). The results are also summarized in Table 3.

\section{Further analysis}

As a post hoc analysis, to control for the confounding effect of statin use, all the chromosomes with LOD/NPL scores $>1.5$ were reanalyzed after subtracting a constant ( $6 \%$ of the sex-specific mean) from the HDL-C values of all statin users. The new results of the quantitative analysis differed somewhat from the previous results, with the new LOD scores being 1.9 for chromosome 2 (2.1 reported previously), 2.9 for chromosome 4 (3.1) 2.8 for chromosome 6 (2.7), 2.4 for chromosome 15 (1.9) and 1.5 for chromosome 17 (2.0). The new results of the qualitative analysis were NPL score 2.0 for chromosome 6 (2.1 reported previously) 1.8 for chromosome 10 (2.3) and 2.6 for chromosome 22 (2.5). The results are also summarized in Table 3. In conclusion, most of the chromosomal regions showing suggestive evidence of linkage in the original analyses also remained suggestive after statin correction, the regions being $4 \mathrm{p} 12$, $6 \mathrm{p} 24,6 \mathrm{p} 12,15 \mathrm{q} 22$ and 22q11.

Chromosome 6, which showed suggestive evidence of linkage in both quantitative and qualitative analysis, was analyzed further. The MAX-TREE statistic of the qualitative analysis resulted in an NPL score of 2.1, suggesting a dominant trait on $6 \mathrm{p} 12$, whereas NPL_ALL, which is most powerful at detecting linkage to an additive trait, yielded a somewhat higher result for $6 \mathrm{p} 22$ than for $6 \mathrm{p} 12$. When we included only overweight subjects $(\mathrm{BMI}>25)$ in the qualitative analysis for the low HDL-C trait, the NPL score on 6p22 was 1.8, whereas after the inclusion of BMI in the QTL analysis, the highest LOD scores were 2.7 (D6S309) and 2.1 (D6S507), for the region 6p2422 , illustrating that the linkage regions of the qualitative and quantitative analysis on chromosome 6 approached each other when BMI was taken into account.

The highest evidence of association in the quantitative association analysis was obtained with marker D6S1713 on 6p25, with a $P$-value of 0.03 . The results of the association analysis with respect to chromosome 6 are summarized in Table 4 .

\section{DISCUSSION}

A low HDL-C level has been found to be the most prevalent dyslipidemia associated with premature $\mathrm{CHD}^{3}$ Given this close relationship, establishment of the genetic background to HDL regulation could illustrate the etiology and pathogenesis of CHD and provide new tools for its prevention and treatment. We undertook a whole-genome scan and detected QTLs for HDL-C regulation showing suggestive evidence of linkage on chromosomes 2q33, 4p12, 6p24 and $17 \mathrm{q} 25$. Three loci for the qualitative low HDL-C trait showing suggestive evidence of linkage were identified in the chromosomal regions $6 \mathrm{p} 12,10 \mathrm{p} 15.3$ and $22 \mathrm{q} 11$. The nearest functional candidate genes for the identified loci are shown in Table 3. Chromosome 2q33 (rs2943634) was significantly associated with CHD in the WTCCC Study and this finding was replicated in the German MI Family Study in a recently published genome-wide association analysis. ${ }^{52}$ Chromosomal locations $6 \mathrm{p} 12-\mathrm{q} 12^{12}$ and $6 \mathrm{p} 22^{10}$ have been linked to HDL-C levels, and an amino acid substitution in the endothelin (EDN1) gene located on 6p24.1 has previously been associated with HDL-C levels in a large analysis of 103 candidate genes for CHD and associated phenotypes in a founder population. ${ }^{53}$ Chromosome 10 has previously shown linkage to HDL-C and TG in other Finnish studies ${ }^{18,54}$ and also for obesity. ${ }^{55}$ Chromosomal region 22q11-q13 has provided suggestive evidence of linkage to HDL-C in a genome-wide scan of serum lipid levels in the Old Order Amish ${ }^{56}$ and in an Australian sample. ${ }^{10}$

PPARD, located on 6p21.2-p21.1, is a nuclear transcription factor regulating lipid metabolism, and its agonists promote reverse cholesterol transport, partly by increasing $A B C A 1$ transcription ${ }^{57}$ and have been shown to increase plasma HDL-C concentrations in insulin-resistant mice ${ }^{58}$ and rhesus monkeys. ${ }^{57} \mathrm{RXRB}$, on $6 \mathrm{p} 21.3$, is a transcription factor that forms heterodimers with oxysterol receptors (liver $X$ receptors, $(L X R s)$ ) and upregulates $A B C A 1$ and apoa-1-mediated cholesterol efflux. ${ }^{59}$ However, the genotyped PPARD and RXRB SNPs showed no statistically significant evidence of association with HDL levels in our sample. As quantitative traits are inherently more informative than disease-health dichotomies, we analyzed the data using the quantitative association test. Marker D6S1713 on 6p25 revealed suggestive evidence of association in the quantitative association analysis

The suggestive QTLs affecting HDL-C variance and suggestive loci for the low HDL-C trait in our sample were partly located on different chromosomes, suggesting that the general variability in HDL-C at the population level may be affected by other genes than those causing the lowest HDL-C levels. It is also important to acknowledge the differences between the two statistical methods and the loss of information because of dichotomizing the trait for the qualitative analysis. We recognize that by analyzing the data using multiple statistics and approaches, there is danger inherent in multiple unadjusted tests, but this concern is at least partly compensated for by the greater power to 
detect linkage with such a complex trait. Although no statistically significant evidence of linkage was observed, it was encouraging that we found suggestive evidence of linkage on seven chromosomal regions. By definition, suggestive evidence of linkage will be found by chance once in each genome scan, and therefore some of these hits should be real and not because of chance. To confirm the results and
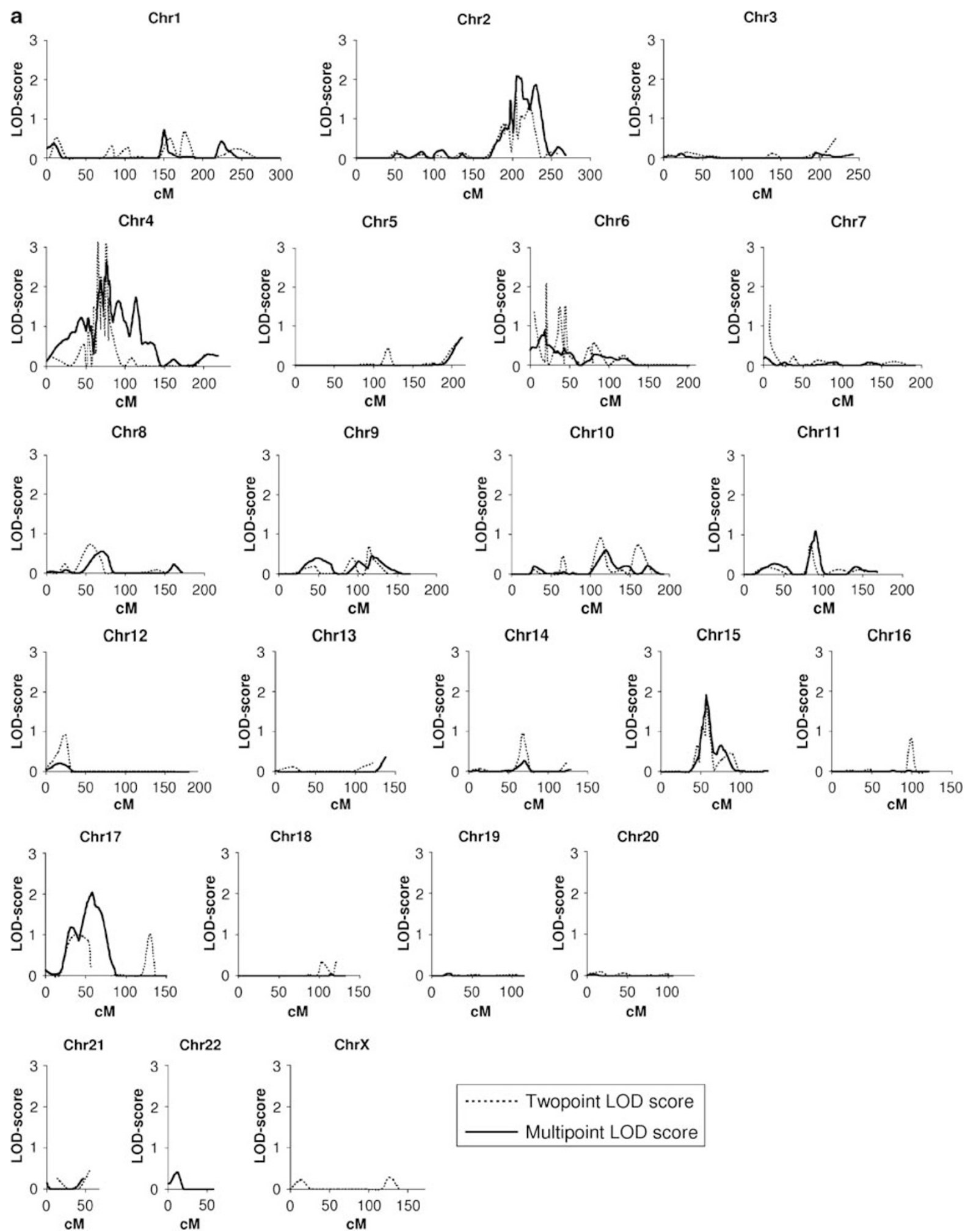

Figure 1 (a) Results of the genome-wide quantitative analysis. All genotyped markers were included in the analysis. The $x$ axis indicates the distance (cM) from the p-terminus and the $y$ axis indicates the LOD score. Chr, chromosome. (b) Results of the genome-wide qualitative analysis. All the statistics calculated before adding markers to the regions with LOD score $\geqslant 1.5$ are presented in the figure. The $x$ axis indicates the distance (cM) from the p-terminus and the $y$ axis indicates the NPL score. Chr, chromosome. 

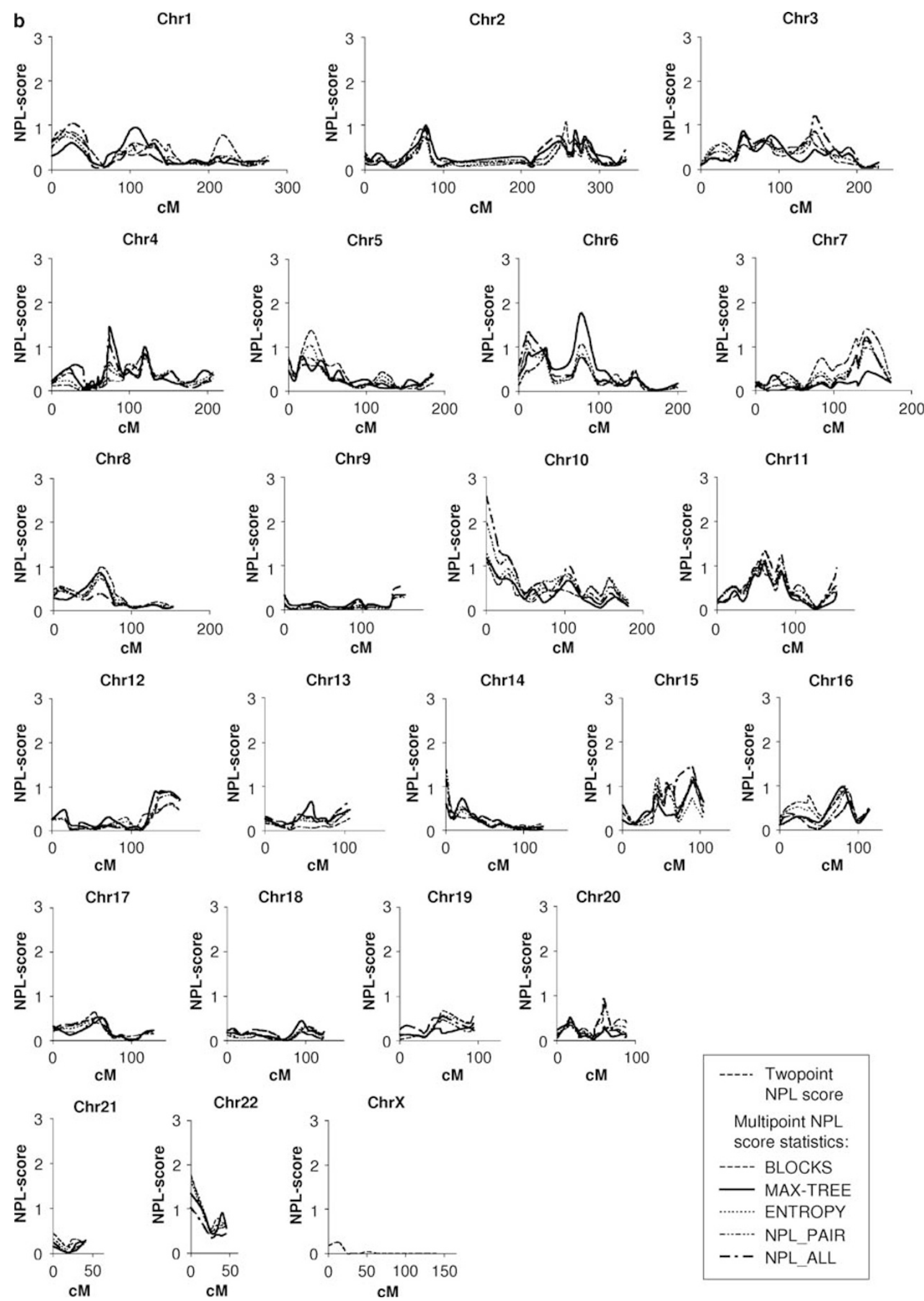

Figure 1 Continued.

to examine the possibility of false-positive signals, these results will need to be replicated in another sample.

The genetic basis of HDL-C regulation seems to be largely heterogeneous. To reduce the heterogeneity and increase the power for finding potential rare variants, our sample was collected from a relatively isolated geographical region and contained large families with probands fulfilling criteria that narrowed the phenotype (the low HDL-C trait associated with early CHD). The linkages revealed in this 

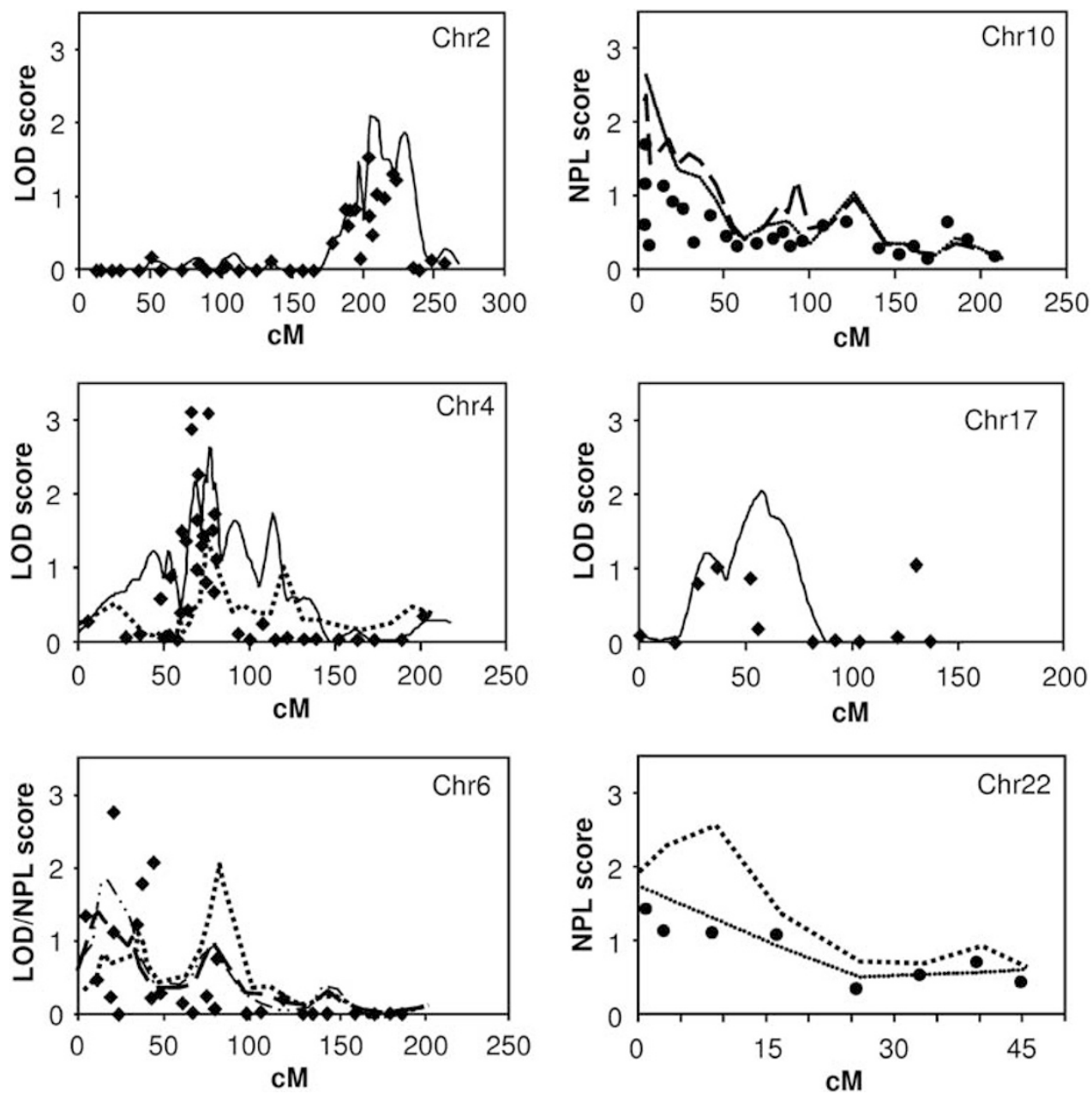

Figure 2 Results showing suggestive evidence of linkage (LOD/NPL score $\geqslant 2.0$ ) in the case of six chromosomes in quantitative analysis (HDL-C as a continuous variable, age and sex as covariates) or in qualitative analysis (subjects having their measured HDL-C levels in the lowest 10th percentile for the general population were coded as affected). Only the results of the MAX-TREE statistics (most powerful at detecting linkage to a dominant trait) or NPL_ALL scores (most powerful at detecting linkage to an additive trait) are reported here for the qualitative analysis, but the results of the other statistics were consistent with these assessments. The $x$ axis indicates the distance (cM) from the first genotyped marker and the $y$ axis indicates the LOD/NPL score. Chr, chromosome. _- quantitative multipoint analysis; —_ - qualitative multipoint analysis, NPL_ALL.................; The same analysis in the initial scan (no additional markers); …..........qualitative multipoint analysis, MAX-TREE;..-- . qualitative multipoint analysis with overweight (BMI $>25)$ subjects with low HDL-C coded as affected, NPL-ALL; $\bullet$ quantitative two-point analysis with sex, age and BMI (for Chr6) as covariates; $\bullet$, qualitative two-point analysis.

study should therefore show evidence of loci affecting the clinically important atherogenic low HDL-C trait. We found evidence of linkage with seven chromosomal regions, the highest having an LOD score of 3.1. Two of the loci have previously shown significant evidence of linkage to HDL-C levels $(6 \mathrm{p} 12)^{12}$ and CHD (2q33). ${ }^{52}$ When taking into account the constant correction for the statin users, the strongest evidence of linkage in the quantitative analysis was detected on chromosomes $4 \mathrm{p} 12$ and $6 \mathrm{p} 24$, and in the qualitative analysis on chromosomes $6 \mathrm{p} 12$ and $22 \mathrm{q} 11$. In addition, in the quantitative analysis, chromosome $15 \mathrm{q} 22$ provided an LOD score exceeding the level of suggestive evidence of linkage only after the constant correction. This same region, including the gene for hepatic lipase (LIPC), has repeatedly shown linkage or association with HDL-C. ${ }^{16,22,23,26-32}$ We cannot exclude the possibility that the linkage signals on chromosomes $17 \mathrm{q} 25$ and $10 \mathrm{p} 15$ could have been false positives originally, as they decreased more than the other cases, in which the changes in LOD/NPL scores because of statin correction were $\pm 0.1-0.2$. From the previously published genome scans for low-HDL-C loci (Tables la and b), the samples of the French Canadian' ${ }^{9}$ and the Finnish studies ${ }^{15,18,25}$ were clinically the most similar to our sample. However, the French Canadian study did not provide information about the CHD status of the subjects and the other Finnish studies included families with familial combined hyperlipidemia (FCHL), making the lipid profile of that sample somewhat different from the current one. The two Finnish scans for low-HDL-C loci have shown significant evidence of linkage to chromosomal regions $8 \mathrm{q} 23^{15}$ and $10 \mathrm{q} 11,{ }^{18}$ which do not overlap with our results. Chromosomal regions 2q21.1-22 and 2q31, which showed significant evidence of linkage to $\mathrm{CHD}^{60}$ and suggestive evidence of linkage to the TG trait ${ }^{54}$ in other Finnish scans, are closer to our finding. Altogether, 18 chromosomes have been described earlier as showing significant evidence for loci regulating HDL-C levels (Tables 1a and $\mathrm{b}$ ). This may at least partly be because of some false-positive signals and the different selection criteria used to recruit the populations, as some samples have been selected on the grounds of CHD, familial hypercholesterolemia, diabetes or hypertension, whereas others have been assembled 
Table 3 Highest LOD scores in the QTL analysis and NPL scores in the nonparametric multipoint linkage analysis for the qualitative low HDL-C trait

\begin{tabular}{|c|c|c|c|c|c|c|}
\hline \multirow[b]{2}{*}{ Location } & \multirow[b]{2}{*}{ Nearest marker } & \multicolumn{2}{|c|}{ Highest $L O D$ score ${ }^{\mathrm{a}}$} & \multicolumn{2}{|c|}{ Highest NPL score ${ }^{\mathrm{b}}$} & \multirow[b]{2}{*}{ Nearest functional candidate genes } \\
\hline & & & Statin use correction ${ }^{\mathrm{C}}$ & & Statin use correction ${ }^{c}$ & \\
\hline 2q33 & D2S155 & 2.1 & 1.9 & & - & ABCA12, HDLBP, LRP2 \\
\hline $4 p 12$ & D4S401 & 3.1 & 2.9 & - & - & PPARGC1A, MTTP \\
\hline \multirow[t]{2}{*}{$6 p 24$} & D6S507 & 2.1 & - & - & - & $\begin{array}{l}\text { PPARD, RXRB, APOM, } \\
\text { EDN1 }\end{array}$ \\
\hline & D6S309 & $2.7^{\mathrm{d}}$ & $2.8^{\mathrm{d}}$ & $1.8^{\mathrm{e}}$ & - & \\
\hline $6 \mathrm{p} 12$ & D6S257 & - & - & 2.1 & 2.0 & \\
\hline 10p15 & D10S249 & - & - & 2.6 & 1.8 & CUBN \\
\hline $15 q 22$ & D15S1033 & 1.9 & 2.4 & - & - & LIPC \\
\hline $17 q 25$ & D17S784 & 2.0 & 1.5 & - & - & $\mathrm{ABCC} 3, \mathrm{ABCA} 5,-6,-9$ and -10 \\
\hline \multirow[t]{2}{*}{$22 q 11$} & D22S427 & - & - & 2.3 & 2.6 & APOL1-6, LRP5L \\
\hline & D22S446 & - & - & 2.5 & 2.6 & \\
\hline
\end{tabular}

Abbreviations: ABCA12, ATP-binding cassette sub-family A member 12; HDLBP, high-density lipoprotein binding protein; LRP2, low-density lipoprotein-related protein 2; PPARGC1A, peroxisome proliferator-activated receptor gamma, coactivator 1 alpha; MTTP, microsomal triglyceride transfer protein; PPARD, peroxisome proliferator-activated receptor-delta; RXRB, retinoid X receptor beta; APOM, apolipoprotein M; EDN1, endothelin 1; CUBN, cubilin; LIPC, hepatic lipase; ABCC3, ATP-binding cassette sub-family C member 3; ABCA5, -6, -9 and -10, ATP-binding cassette subfamily A members 5, 6, 9 and 10; APOL1-6, apolipoproteins L1-L6, LRP5L, low-density lipoprotein receptor-related protein 5-like. Only LOD/NPL scores $\geqslant 1.5$ are shown for chromosomes with at least one score $\geqslant 2.0$

aLOD scores in the QTL analysis (HDL-C as a continuous variable, age and sex as covariates).

${ }^{b}$ Multipoint NPL scores in the low HDL-C-trait analysis (subjects having their measured HDL-C levels in the lowest 10th percentile for the general population were coded as affected).

cTo control the statin use as a possible confounding factor, the chromosomes with LOD/NPL score $>1.5$ were re-analyzed with a constant $(6 \%$ of the mean HDL-C levels: $0.06 \mathrm{mmol}$ for men and

$0.09 \mathrm{mmol}$ for women) subtracted from HDL-C values of the statin users.

${ }^{\mathrm{d}}$ Age, sex and BMI as covariates.

e Multipoint analysis in which low HDL-C patients with BMI $>25$ were considered affected.

Table $4 P$-values for the quantitative association analysis

\begin{tabular}{|c|c|c|}
\hline Marker/gene & Location (Mb) & Quantitative association test \\
\hline D6S344 & 1.55 & 0.821 \\
\hline D6S1713 & 4.29 & 0.032 \\
\hline D6S1640 & 7.35 & 0.863 \\
\hline D6S309 & 8.17 & 0.301 \\
\hline D6S263 & 8.49 & 0.237 \\
\hline D6S470 & 10.13 & 0.943 \\
\hline D6S289 & 15.39 & 0.325 \\
\hline D6S1584 & 16.82 & 0.972 \\
\hline D6S422 & 20.48 & 0.415 \\
\hline D6S507 & 21.92 & 0.095 \\
\hline D6S276 & 24.29 & 0.677 \\
\hline rs6531/RXRB & 33.27 & 0.451 \\
\hline rs2076310/RXRB & 33.27 & 0.140 \\
\hline rs1547387/RXRB & 33.28 & 0.613 \\
\hline rs11751895/PPARD & 35.44 & 0.176 \\
\hline rs9470001/PPARD & 35.44 & 0.607 \\
\hline rs1883322/PPARD & 35.48 & 0.096 \\
\hline rs9658130/PPARD & 35.49 & 0.629 \\
\hline rs2076169/PPARD & 35.50 & 0.313 \\
\hline D6S426 & 40.75 & 0.662 \\
\hline D6S271 & 43.61 & 0.221 \\
\hline D6S1714 & 51.66 & 0.228 \\
\hline D6S257 & 56.03 & 0.207 \\
\hline D6S1711 & 62.60 & 0.340 \\
\hline
\end{tabular}

Abbreviations: Link. Linkage. LD linkage disequilibrium. $P$-values for the quantitative association analysis in the linkage region 6 p25-6q11.1 are presented in the table. $P$-values of $<0.05$ are shown in bold. In the quantitative analysis, HDL-C was used as a continuous trait.

randomly. Because of the small number of original founders, the isolation of the Finnish population for the past centuries and genetic drift, the loci for HDL-C regulation in the homogeneous Finnish population, or even in regional subpopulations, might be specific and somewhat unique, ${ }^{61,62}$ whereas they could be of minor importance in other, more heterogeneous, populations. Their identification would nevertheless provide important knowledge about HDL metabolism and the relationship between HDL and atherogenesis.

\section{CONFLICT OF INTEREST}

The authors declare no conflict of interest.

\section{ACKNOWLEDGEMENTS}

We acknowledge the excellent technical assistance provided by Ms Marja-Leena Kytökangas, Ms Saara Korhonen, Ms Saija Kortetjärvi, Ms Sari Pyrhönen and Ms Sirpa Rannikko. Doctors Jukka Juvonen, Tuomo Jääskeläinen, Erkki Kiviniemi and Mikko Lehtola (deceased) are greatly acknowledged for their help in collecting the pedigrees. Dr Thomas Dyer and Dr Laura Almasy are acknowledged for their valuable counseling on SOLAR usage. This work was supported by grants from the Finnish Foundation for Cardiovascular Research, the Finnish Cultural Foundation, the Finnish Medical Society Duodecim, the Finnish Medical Foundation, the Medical Council of the Academy of Finland, the Paavo Nurmi Foundation, the Sigrid Jusélius Foundation, the Onni and Hilja Tuovinen Foundation and the US National Institutes of Health.

1 Lusis AJ: Atherosclerosis. Nature 2000; 6801: 233-241.

2 Gordon T, Castelli WP, Hjortland MC, Kannel WB, Dawber TR: High density lipoprotein as a protective factor against coronary heart disease. The Framingham Study. Am J Med 1977; 5: 707-714.

3 Genest Jr JJ, Martin-Munley SS, McNamara JR et al: Familial lipoprotein disorders in patients with premature coronary artery disease. Circulation 1992; 6: 2025-2033.

4 Wang X, Paigen B: Genetics of variation in HDL cholesterol in humans and mice. Circ Res 2005; 1: 27-42.

5 Kullo IJ, Turner ST, Boerwinkle E, Kardia SL, de AM: A novel quantitative trait locus on chromosome 1 with pleiotropic effects on HDL-cholesterol and LDL particle size in hypertensive sibships. Am J Hypertens 2005; 8: 1084-1090.

6 Arya R, Lehman D, Hunt KJ et al: Evidence for bivariate linkage of obesity and HDL-C levels in the Framingham Heart Study. BMC Genet 2003; S52.

7 Gagnon F, Jarvik GP, Badzioch MD, Motulsky AG, Brunzell JD, Wijsman EM: Genome scan for quantitative trait loci influencing HDL levels: evidence for multilocus inheritance in familial combined hyperlipidemia. Hum Genet 2005; 117: 494-505. 
8 Bielinski SJ, Tang W, Pankow JS et al: Genome-wide linkage scans for loci affecting total cholesterol, HDL-C, and triglycerides: the Family Blood Pressure Program. Hum Genet 2006; 3: 371-380.

9 Dastani Z, Quiogue L, Plaisier C et al: Evidence for a gene influencing high-density lipoprotein cholesterol on chromosome 4q31.21. Arterioscler Thromb Vasc Biol 2006; 2: 392-397.

10 Harrap SB, Wong ZY, Scurrah KJ, Lamantia A: Genome-wide linkage analysis of population variation in high-density lipoprotein cholesterol. Hum Genet 2006; 119: 541-546.

11 Peacock JM, Arnett DK, Atwood LD et al: Genome scan for quantitative trait loci linked to high-density lipoprotein cholesterol: the NHLBI Family Heart Study. Arterioscler Thromb Vasc Biol 2001; 11: 1823-1828.

12 Canizales-Quinteros S, uilar-Salinas CA, Reyes-Rodriguez E et al: Locus on chromosome $6 p$ linked to elevated HDL cholesterol serum levels and to protection against premature atherosclerosis in a kindred with familial hypercholesterolemia. Circ Res 2003; 5: 569-576.

13 Yang $Q$, Lai $C Q$, Parnell $L$ et al: Genome-wide linkage analyses and candidate gene fine mapping for HDL3 cholesterol: the Framingham Study. J Lipid Res 2005; 7: 1416-1425.

14 Adeyemo AA, Johnson T, Acheampong J et al: A genome wide quantitative trait linkage analysis for serum lipids in type 2 diabetes in an African population. Atherosclerosis 2005; 2: 389-397.

15 Soro A, Pajukanta P, Lilja HE et al: Genome scans provide evidence for low-HDL-C loci on chromosomes 8q23, 16q24.1-24.2, and 20q13.11 in Finnish families. Am J Hum Genet 2002; 5: 1333-1340.

16 Almasy L, Hixson JE, Rainwater DL et al: Human pedigree-based quantitative-traitlocus mapping: localization of two genes influencing HDL-cholesterol metabolism. Am J Hum Genet 1999; 6: 1686-1693.

17 Arya R, Duggirala R, Almasy L et al: Linkage of high-density lipoprotein-cholesterol concentrations to a locus on chromosome 9p in Mexican Americans. Nat Genet 2002. 1: 102-105

18 Lilja HE, Suviolahti E, Soro-Paavonen A et al: Locus for quantitative HDL-cholesterol on chromosome 10q in Finnish families with dyslipidemia. J Lipid Res 2004; 10 1876-1884.

19 Bosse Y, Chagnon YC, Despres JP et al: Genome-wide linkage scan reveals multiple susceptibility loci influencing lipid and lipoprotein levels in the Quebec family study. J Lipid Res 2004; 3: 419-426.

20 Hsiao CF, Chiu YF, Chiang FT et al: Genome-wide linkage analysis of lipids in nondiabetic Chinese and Japanese from the SAPPHIRe family study. Am J Hypertens 2006; 12: 1270-1277

21 Feitosa ME, Rice T, Borecki IB et al: Pleiotropic QTL on chromosome 12q23-q24 influences triglyceride and high-density lipoprotein cholesterol levels: the HERITAGE family study. Hum Biol 2006; 3: 317-327.

22 Feitosa MF, Province MA, Heiss G et al: Evidence of QTL on $15 q 21$ for high-density lipoprotein cholesterol: the National Heart, Lung, and Blood Institute Family Heart Study (NHLBI FHS). Atherosclerosis 2007; 1: 232-237.

23 Yu Y, Wyszynski DF, Waterworth DM et al: Multiple QTLs influencing triglyceride and $\mathrm{HDL}$ and total cholesterol levels identified in families with atherogenic dyslipidemia. J Lipid Res 2005; 10: 2202-2213.

24 Mahaney MC, Almasy L, Rainwater DL et al: A quantitative trait locus on chromosome $16 q$ influences variation in plasma HDL-C levels in Mexican Americans. Arterioscle Thromb Vasc Biol 2003; 2: 339-345.

25 Pajukanta P, Allayee H, Krass KL et al: Combined analysis of genome scans of Dutch and Finnish families reveals a susceptibility locus for high-density lipoprotein cholesterol on chromosome 16q. Am J Hum Genet 2003; 4: 903-917.

26 Kathiresan S, Willer CJ, Peloso GM et al: Common variants at 30 loci contribute to polygenic dyslipidemia. Nat Genet 2009; 1: 56-65.

27 Willer CJ, Sanna S, Jackson AU et al: Newly identified loci that influence lipid concentrations and risk of coronary artery disease. Nat Genet 2008; 2: 161-169.

28 Aulchenko YS, Ripatti S, Lindqvist I et al: Loci influencing lipid levels and coronary heart disease risk in 16 European population cohorts. Nat Genet 2009; 1: 47-55.

29 Kooner JS, Chambers JC, Guilar-Salinas CA et al: Genome-wide scan identifies variation in MLXIPL associated with plasma triglycerides. Nat Genet 2008; 2: 149-151.

30 Saxena R, Voight BF, Lyssenko V et al: Genome-wide association analysis identifies loci for type 2 diabetes and triglyceride levels. Science 2007. 5829. 1331-1336.

31 Wallace C, Newhouse SJ, Braund P et al: Genome-wide association study identifies genes for biomarkers of cardiovascular disease: serum urate and dyslipidemia. $\mathrm{Am} \mathrm{J}$ Hum Genet 2008; 1: 139-149.

32 Sabatti C, Service SK, Hartikainen AL et al: Genome-wide association analysis of metabolic traits in a birth cohort from a founder population. Nat Genet 2009; 1: 35-46.

33 Sirtori CR, Calabresi L, Franceschini G et al: Cardiovascular status of carriers of the apolipoprotein A-I(Milano) mutant: the Limone sul Garda study. Circulation 2001; 15: 1949-1954.
34 Jansen $\mathrm{H}$, Verhoeven AJ, Weeks L et al: Common C-to-T substitution at position -480 of the hepatic lipase promoter associated with a lowered lipase activity in coronary artery disease patients. Arterioscler Thromb Vasc Biol 1997; 11: 2837-2842.

35 Peltonen L, Jalanko A, Varilo T: Molecular genetics of the Finnish disease heritage. Hum Mol Genet 1999; 10: 1913-1923.

36 Jones PH, Davidson MH, Stein EA et al: Comparison of the efficacy and safety of rosuvastatin versus atorvastatin, simvastatin, and pravastatin across doses (STEL$\mathrm{LAR}^{*}$ Trial). Am J Cardiol 2003; 2: 152-160.

37 Tobin MD, Sheehan NA, Scurrah KJ, Burton PR: Adjusting for treatment effects in studies of quantitative traits: antihypertensive therapy and systolic blood pressure. Stat Med 2005; 19: 2911-2935

38 Rantala AO, Kauma H, Lilja M, Savolainen MJ, Reunanen A, Kesäniemi YA: Prevalence of the metabolic syndrome in drug-treated hypertensive patients and control subjects. J Intern Med 1999; 2: 163-174.

$39 \mathrm{Dib}$ C, Faure S, Fizames $\mathrm{C}$ et al: A comprehensive genetic map of the human genome based on 5264 microsatellites. Nature 1996; 6570: 152-154.

40 Kakko S, Kelloniemi J, von Rohr P et al: ATP-binding cassette transporter A1 locus is not a major determinant of HDL-C levels in a population at high risk for coronary heart disease. Atherosclerosis 2003; 2: 285-290.

41 Abecasis GR, Cherny SS, Cookson WO, Cardon LR: GRR: graphical representation of relationship errors. Bioinformatics 2001; 8: 742-743.

42 O'connell JR, Weeks DE: PedCheck: a program for identification of genotype incompatibilities in linkage analysis. Am J Hum Genet 1998; 1: 259-266.

$43 \mathrm{Amos} \mathrm{Cl}$ : Robust variance-components approach for assessing genetic linkage in pedigrees. Am J Hum Genet 1994; 3: 535-543.

44 Almasy L, Blangero J: Multipoint quantitative-trait linkage analysis in general pedigrees. Am J Hum Genet 1998; 5: 1198-1211.

45 Sobel E, Lange K: Descent graphs in pedigree analysis: applications to haplotyping, location scores, and marker-sharing statistics. Am J Hum Genet 1996; 6: 1323-1337.

46 Abecasis GR, Cherny SS, Cookson WO, Cardon LR: Merlin-rapid analysis of dense genetic maps using sparse gene flow trees. Nat Genet 2002; 1: 97-101.

47 Terwilliger JD, Goring HH: Gene mapping in the 20th and 21st centuries: statistical methods, data analysis, and experimental design. Hum Biol 2000; 1: 63-132.

48 Mukhopadhyay N, Almasy L, Schroeder M, Mulvihill WP, Weeks DE: Mega2: datahandling for facilitating genetic linkage and association analyses. Bioinformatics 2005; 10: 2556-2557.

49 Lange EM, Lange K: Powerful allele sharing statistics for nonparametric linkage analysis. Hum Hered 2004; 1: 49-58.

50 Lange K, Cantor R, Horvath S et al: Mendel version 4.0: A complete package for the exact genetic analysis of discrete traits in pedigree and population data sets. $\mathrm{Am} \mathrm{J}$ Hum Genet 2001; 69 (Suppl): A1886.

51 Lander E, Kruglyak L: Genetic dissection of complex traits: guidelines for interpreting and reporting linkage results. Nat Genet 1995; 3: 241-247.

52 Samani NJ, Erdmann J, Hall AS et al: Genomewide association analysis of coronary artery disease. N Eng/ J Med 2007; 5: 443-453.

53 Pare G, Serre D, Brisson D et al: Genetic analysis of 103 candidate genes for coronary artery disease and associated phenotypes in a founder population reveals a new association between endothelin-1 and high-density lipoprotein cholesterol. Am J Hum Genet 2007; 4: 673-682.

54 Pajukanta P, Terwilliger JD, Perola M et al: Genomewide scan for familial combined hyperlipidemia genes in Finnish families, suggesting multiple susceptibility loci influencing triglyceride, cholesterol, and apolipoprotein B levels. Am J Hum Genet 1999; 5: 1453-1463.

55 Hager J, Dina C, Francke S et al: A genome-wide scan for human obesity genes reveals a major susceptibility locus on chromosome 10. Nat Genet 1998; 3: 304-308.

56 Pollin TI, Hsueh WC, Steinle NI, Snitker S, Shuldiner AR, Mitchell BD: A genomewide scan of serum lipid levels in the Old Order Amish. Atherosclerosis 2004; 1: 89-96.

57 Oliver Jr WR, Shenk JL, Snaith MR et al: A selective peroxisome proliferator-activated receptor delta agonist promotes reverse cholesterol transport. Proc Natl Acad Sci USA 2001; 9: 5306-5311.

58 Leibowitz MD, Fievet $\mathrm{C}$, Hennuyer $\mathrm{N}$ et al: Activation of PPARdelta alters lipid metabolism in db/db mice. FEBS Lett 2000; 3: 333-336.

59 Schwartz K, Lawn RM, Wade DP: ABC1 gene expression and ApoA-I-mediated cholesterol efflux are regulated by LXR. Biochem Biophys Res Commun 2000; 3: 794-802.

60 Pajukanta P, Cargill M, Viitanen L et al: Two loci on chromosomes 2 and X for premature coronary heart disease identified in early- and late-settlement populations of Finland. Am J Hum Genet 2000; 6: 1481-1493.

61 Varilo T, Peltonen L: Isolates and their potential use in complex gene mapping efforts. Curr Opin Genet Dev 2004; 3: 316-323.

62 Service S, DeYoung J, Karayiorgou M et al: Magnitude and distribution of linkage disequilibrium in population isolates and implications for genome-wide association studies. Nat Genet 2006; 5: 556-560. 